Zoologischen Museums statt, der ein Besuch der Bernsteinsammlung der Universität folgte. Der Verwalter der Sammlung gab die zum Verständnis der Sammlungen notwendigen Mitteilungen über Entstehung, Farben und Gewinnung des Bernsteins, sowie über Bernstein-Einschlüsse, unter denen sich auch vier mit Vogelfedern oder Teilen von solchen befanden.

Den übrigen Teil des Nachmittags wurde der Besichtigung des „Königsberger Tiergartens“ unter Führung des Herrn Geheimrat Kla a ss gewidmet.

Die grofse Ausdehnung des an landschaftlichen Reizen reichen Gartens ermöglicht es, den Tieren grofse Räume zu geben. Die grofsen Flugkäfige für Raubvögel, die in bügeligem Gelände erbaute, von einem Bach durchflossene grofse Voliere für Möwen, Kormorane, Reiher und verwandte Arten, das Haus für Kleinvögel sind besonders erwähnenswert. Ausgezeichnet ist das Aussehen der Tiere, grofs die Zahl der Züchtungen. Dank der tüchtigen Verwaltung des Gartens und der Unterstützung durch staatliche und städtische Behörden ist es ermöglicht, in eigenem landwirtschaftlichem Betrieb einen grofsen Teil der Futtermittel den Tieren in dem der Jahreszeit entsprechenden Entwicklungszustand zu bieten.

Über die Vorteile einer derartigen Fütterung und die Verbindung eines zoologischen Gartens mit einem landwirtschaftlichen Betrieb teilte Herr Geheimrat Klaass Näheres gelegentlich des gemeinsamen Essens, welches im „Tiergarten“ stattfand, mit. Es war das letzte programmgemäfse Beisammensein der Teilnehmer an der aufserordentlich gelungenen Jahresversammlung der ,deutschen ornithologischen Gesellschaft" im Jahre 1908.
O. Heinroth.
K. Neunzig.

\title{
Über den gegenwärtigen Stand der Naumannforschung.
}

Bericht von

\section{Herman Schalow.}

Auf allen Gebieten geistigen Schaffens, wo es sich auch immer in den Dienst der Erweiterung unserer schwachen menschlichen Erkenntnis stellt, begegnen wir der Tatsache, dafs zeitweilig Männer auftreten, deren Arbeiten einen Merkstein für eine bestimmte Epoche wissenschaftlicher Forschung darstellen, oft aber auch einen Schlufsstein innerhalb derselben bilden. Langes Tasten und Suchen kommt endgültig zum Abschluss. Die von solchen Männern gewonnenen Ergebnisse, im einzelnen vielleicht anfechtbar, bilden das Fundament zu späterem Ausbau. Ihre Arbeit regt zu weiterer Forschung an und weckt die schlummernden Kräfte. Eine neue umfassende Anschaunngsweise gelangt zum

Journ. f. Orn. LVI. Jahrg. Januar 1909. 
Ausdruck. Die Bedeutung derselben beruht nicht nur auf dem Werte, welchen sie an und für sich besitzt, sondern auch in dem Einflufs, den sie auf die Nebenzweige der betreffenden Wissenschaft ausübt.

Für unsere deutsche Ornithologie ist Joh. Fr. Naumann ein solcher Bahnbrecher. Er hat Neues geschaffen; er hat durch seine hervorragenden wissenschaftlichen Leistungen und durch ein energisches Wollen ein hohes Ziel erreicht. Naumann war der Mann, der in rastloser Arbeit allmählich das gesamte Gebiet der Lebensgeschichte unserer deutschen Vögel durch exacte Beobachtungen in mustergültigster Weise förderte und festlegte. Er war der berufene Vertreter einer Richtung in der Ornithologie, von der manche damals schon meinten, dass sie sich ausgelebt habe, und dafs ein innerer Wert ihr nicht innewohne, von der wir heute aber wissen, dafs wir eben anfangen in sie einzudringen. Als Naumann am 15. August 1857 in Ziebigk, in demselben Hause, in dem er geboren, die Augen schlofs, verlor die ornithologische Wissenschaft einen Jünger der ausgeprägtesten Individualität und von seltener Vielseitigkeit. Durch die überaus glückliche Vereinigung scharfer Beobachtungsgabe, starken zielbewufsten Willens und künstlerischer Veranlagung verlieh er seinem grofsen unsterblichen Werke eine specielle Signatur. In unserer Zeit, der eine über das Mafs des Notwendigen hinausgehende Specialisierung nicht mit Unrecht zum Vorwurf gemacht wird, wirkt der Rückblick auf Naumann's Erscheinung, die bei tiefem Eindringen in Einzelfragen der individuellen Biologie nie den Blick auf das Gesamtgebiet verliert, doppelt sympatisch. Johann Friedrich Naumann war Doctor honoris causa, Träger hoher Orden und Professor gar; aber er war nach meinem Empfinden mehr als Alles das: ein allseitig entwickelter Mensch und ein charakterfester Mann, zwei Eigenschaften, die in unserer Zeit des Specialistentums und des Opportunismus allmählich selten zu werden beginnen. In dem Grundzug seines Wesens war, nach den Mitteilungen seiner Zeitgenossen, eine von tiefer Herzensbildung zeugende Milde der Gesinnung. Sie ward getragen von einem Idealismus, der nur Naturen von tiefem inneren Wesen eigen $\mathrm{zu}$ sein pflegt. Mit tüchtigen und trefflichen Männern hat er langjährige Freundschaft gepflegt. Er erkannte seine Leute und er kannte das Leben. Seine Polemik war oft scharf, wie uns $\mathrm{Baldamus}$ ) berichtet, aber sie richtete sich stets gegen die Sache, nie gegen die Person. Naumann hatte sich eine umfassende Bildung erworben; auf den verschiedensten Gebieten wissenschaftlicher Arbeit vermochte er den Fortschritten seiner Zeit zu folgen und sich Rechenschaft über die Aufgaben zu geben, die sie beschäftigte. Sein Kunstinteresse galt vornehmlich der Musik.

1) Bald a mu s, Ed., Die drei Naumanns; Daheim, 1866, S. $332-337$. 
Die langen Winterabende in der ländlichen Einsamkeit waren ihr gewidmet. Sie verschönte ihm und den Seinen die Stunden der Ruhe. Denn als ein wahrhaft cultivierter Mensch konnte und wollte er der Mufse nicht entbehren.

Johann Friedrich Naumann's Leben ist den weitesten Umrissen nach allgemein bekannt. Was in engem Rahmen über dasselbe zu sagen war, ist von berufener, oft auch unberufener Seite geschehen. Mehr von unberufener. Fast Alle, die es geschildert, folgen den autobiographischen Mitteilungen, welche der Vater, Johann Andreas Naumann, in der ersten Auflage seiner in Octav erschienenen ausführlichen Beschreibung aller Wald-, Feld- und Wasservögel im Jahre 1797 gab, und die der Sohn Johann Friedrich im ersten Teile seiner 12 bändigen Naturgeschichte, 1820, wieder abdruckte Eigene Quellenstudien, zu denen doch mancherlei Material anregte, fehlen fast ganz. Ueber Naumann's phänomenales Werk selbst herrscht beiallen Beurtheilern nur eine Stimme. Es sei gestattet, die Worte zu wiederholen, die ich im Jahre 1893, gelegentlich einer Versammlung unserer Gesellschaft in Cassel, über dasselbe sagte. ${ }^{\mathbf{1}}$ )

„Im Jahre 1844 liefs Johann Friedrich Naumann den zwölften Band seiner Naturgeschichte der Vögel Deutschlands erscheinen. Dieser Band bildet den Abschlufs einer Arbeit, die der grofse deutsche Ornithologe als seine Lebensaufgabe bezeichnet hat, deren Lösung er, in zielbewufstem Streben, mehr denn fünfzig Jahre emsigster und eingehendster Tätigkeit gewidmet hatte. Allüberall und rückhaltlos ist Naumanus Werk als ein klassisches anerkannt worden, nicht nur in unserem engeren Vaterlande, sondern überall, wo man den Lebenserscheinungen in der Welt der Vögel ein eingehendes Studium widmet. Oft ist gegen die Schilderungen Naumanns der Vorwurf erhoben worden, dafs dieselben in allzu behaglicher Breite und Ausführung den Gegenstand behandeln, und dafs die Darstellung vielfach des einheitlichen Charakters entbehre. Ja, ein gewisses Ringen nach scharfem, präcisen Ausdruck, ein Suchen nach dem passendem Wort, ein breitgefügter Periodenbau sind dem Stile Naumanns nachgesagt worden. Diese Vorwürfe gegen die Darstellung sind nicht ohne jede Berechtigung. Allein in dem ganzen Bildungsgange, in der ganzen Entwicklung der wissenschaftlichen Tätigkeit unseres grofsen vaterländischen Ornithologen, der es als Autodidact dahin gebracht, von den Fachmännern als ebenbürtig anerkannt zu werden, liegt der erklärliche Grund und zugleich die natürliche Entschuldigung für diese Erscheinung. Naumann besafs in hervorragendem Sinne das, was Rolsmäfsler als erste Bedingung bezeichnet, um sich, sowohl für sich selbst wie für die Wissenschaft, zum Naturforscher herauszubilden: er besafs fünf gesunde Sinne, ein nüchternes, scharfes Urteil, verbunden mit einem treuen Gedächtnis,

1) Journ. f. Ornith. 1894, 270-280. 
und eine an Verehrung streifende Liebe für die ihn umgebende Natur. Er war kein systematischer Kopf und viel Generalisieren nicht seine Sache. Alles, was er gab, schuf er aus sich selbst; in der Beschreibung des Lebens unserer deutschen Vögel war er ohne eigentlichen Vorgänger. Ich glaube, dafs die behagliche Breite der biologischen Darstellung bei Naumann, abgesehen von sicherlich individueller Anlage, zum überwiegend gröfsten Teil auf eine ängstliche Besorgnis zurückzuführen ist, in möglichster Vollständigkeit, bei scrupulösester Wahrheitsliebe und Gewissenhaftigkeit, jeden Zug, wenn ich so sagen darf, eine jede Lebenserscheinung des einźelnen Vogels, wie er sie durch eine Unsumme jahrelang fortgesetzter, einzelner, kleiner Beobachtungen fixiert hatte, peinlich so wiederzugeben, das auch nicht das unbedeutenste Moment dabei verloren ginge. Und von diesem Gesichtspunkte aus betrachtet müssen wir die Naumann'sche Darstellung des Lebens unserer heimischen Vögel auch heute noch als einfach mustergültige, als eine klassische bezeichnen."

Überall teilt man diese meine Ansicht, auch aufserhalb Deutschlands. Henry Seebohm ${ }^{1}$ ) bezeichnet Naumanns Arbeit als ein Werk ohne Rivalen in Vergangenheit, Gegenwart und Zukunft. Alfred Newton ${ }^{2}$ ) veröffentlichte gelegentlich des Abschlusses des „,neuen Naumann" im Jahre 1905 in der Nature einen Aufsatz, in welchem er $u$. a. von dem alten Werke Naumanns sagt, dafs ,kein Werk, die Vollständigkeit des Inhalts angehend, in irgend einer Sprache existiere, das ihm gleiche“. Naumann schrieb, führt Newton aus, nach seinen eigenen Erfahrungen und Beobachtungen; sein Wissen war gesund: er hatte nicht nötig von Jemandem etwas zu borgen.

Ein Mann von der hohen Bedeutung Naumann's für die deutsche Ornithologie verdient ein literarisches Denkmal, zu seiner Ehre, zum Nutzen der Wissenschaft, zum Ansporn für die ornithologische Nachwelt. In seinem Vaterlande Amerika hat man ein solches Audubon ${ }^{3}$ ) längst errichtet; Otto Herman ${ }^{4}$ ) hat in seiner fortreifsenden Begeisterung seinen Landsmann Janos Petényi in einer wundervollen Monographie gefeiert; und

1) Henry Se ebo hm, A History of British Birds, vol. III p. XlI. (1885).

$\left.{ }^{2}\right)$ Alfred Newton, The Naumann Festival at Cöthen; The Nature 1905, May/Oct. p. 36-37; Vergl. R. Blasius, Ornith. Monatsschrift 1905, $400-404$.

3) Horace St. John, Audubon, the naturalist in the new world: his adventures and discoveries. London 8; Longman, Brown, Green and Longmans, 1856, XIV + 172 .

R. Buchanan, The life and adventures of J. J. Audubon the naturalist. II. Aufl. New York and London 1869.

4) 0 tto Herman, J. S. von Petényi, der Begründer der wissenschaftlichen Ornithologie in Ungarn. 1799-1855. Budapest 1891. fol. $137 \mathrm{pp}$. 
von dem bekannten Germanisten Ludwig Bechstein ${ }^{1}$ ) ist die Erinnerung an Joh. Matthäus Bechstein in einem umfangreichen Quellenwerk festgehalten worden, welches von den ornithologischen Bibliophilen hochgeschätzt wird. Wie steht es nun mit einer Naumann Biographie? Jean Cabanis schrieb mir in dem letzten Briefe, den ich von seiner Hand besitze: „Es hat mir immer leid gethan, dafs in unserer ornithologischen Wissenschaft so wenig Sinn für die Biographie und für die historische Entwicklung der Ornithologie vorhanden war." Die Wahrheit dieser Bemerkung habe ich nie mehr empfunden als im Hinblick auf die Frage nach dem Stande unserer Naumann Forschung. Aufser den bereits genannten autobiographischen Aufzeichnungen und dem ausgezeichneten Vorwort, mit reichen bibliographischen Einzelheiten, gewonnen auf Grund sorgfältiger Quellenstudien, welches Paul Leverkühn für den „neuen Naumann" geschrieben, besitzen wir vorläufig nichts im Druck Vorliegendes, das man als eine u mfassende Naumann Biographie bezeichnen könnte. Dabei harrt schon jetzt ein, wenn auch nicht grosses, so doch wertvolles Material der Bearbeitung. Nur verhältnismäfsig wenige Briefe scheint ein günstiger Zufall in treue Hände gebracht $\mathrm{zu}$ haben. Unser Mitglied Jacob Moyat in Mainz besitzt etwas; ein anderer Teil des Briefwechsels soll sich in den Händen Leverkühns befunden haben. Hierunter vielleicht auch die an Baldamus gerichteten Briefe. Eugen von Homeyer ${ }^{2}$ ) erwähnt in seinem Buch nur dreier Schreiben von Naumann. Alles wäre zu sichten. Es wäre interessant, aus dem Briefwechsel $u$. a. die Beziehungen unseres grofsen Ornithologen zu seinem Leipziger Verleger Gerhard Fleischer $\mathrm{zu}$ erörtern und die finanziellen Opfer kennen zu lernen, die er sicherlich in damaliger Zeit der Herausgabe seines grofsen Werkes gebracht haben wird. Die Beziehungen Naumann's zu den Ornithologen seiner Zeit könnten untersucht und ferner dargestellt werden, ob, wann und wie die ausländischen Vogelkundigen und Fachzeitschriften von ihm und seinem Werke Notiz nehmen. Wir besitzen im Augenblick keine erschöpfende Bibliographie seiner Arbeiten. Aus den in seiner Bibliothek befindlichen Büchern, die sich noch heute in seinem Wohnhause in Ziebigk befinden, würden sich mancherlei Gesichtspunkte für die Beurteilung der Persönlichkeit Naumann's ergeben. Denn für ihn waren seine Bücher kein totes Capital. An seiner Bibliothek haftet ein Stück seiner geistigen Entwickelung. Daher wird in einer Biographie Naumanns der Nachweis von hohem Interesse sein, wie die feinen Fäden von fremden Büchern $\mathrm{zu}$ seinen eigenen Arbeiten hinüberführen. Besonderer Wert liegt auch

1) Ludwig Bechstein, Dr. Johann Matthäus Bechstein u. die Forstakademie Dreifsigacker. Meiningen 1855. gr. 8. 420 pp.

$\left.{ }^{2}\right)$ Eugen von Homeyer, Ornithologische Briefe. Blatter der Erinnerung an seine Freunde. Berlin 1881, 80, VI + 340 pp. [32-36]. 
in den Einzeichnungen, die Naumann auf den durchschossenen Blättern einzelner Bücher einzutragen liebte. Auch die handschriftlichen Dedikationen bieten ein Interesse. Die ersten Versuche und die spätere Entwickelung Naumann's als Zeichner und Kupferstecher wären zu schildern, und was dergleichen Tatsachen mehr sind, die zusammen dann ein Bild des grofsen Gelehrten, wie es der Nachwelt erhalten werden mufs, geben sollen. Eine getreue Darstellung des Lebens und Schaffens bedeutender Menschen läfst sich nur auf der Grundlage der Kenntnis und der sorgsamen Betrachtung ihrer sämtlichen Werke und ihres gesamten Tuns gewinnen.

Als ich vor langen Jahren, 1880, in Gemeinschaft mit meinem verstorbenen Freunde Léon Olphe-Galliard in Lyon das Lebensbild eines französischen Ornithologen zu zeichnen suchte ${ }^{1}$ ) wurde ich auf die manigfaltigen Ähnlichkeiten aufmerksam, welche sich in dem Leben von Jean Crespon und dem J. Fr. Naumanns darboten. Beide Autodidacten. Der eine Friseur, Fecht- und Tanzlehrer, der andere ein einfacher Landwirt. Und Beide haben sie auf dem Gebiete der Ornithologie Grofses geschaffen. Als ich mich mit Studien über den eigenartigen Lebensgang Crespons beschäftigte wurde ich bereits damals mehr und mehr von der Notwendigkeit der Herausgabe einer umfassenden Biographie unseres grofsen Landsmannes durchdrungen. In Niemandes Hand konnte diese Arbeit besser gelegt werden, als in diejenige unseres tief beklagten Freundes Paul Leverkühn. Er hatte es unternommen, Joh. Fr. Naumann ein literarisches Denkmal zu errichten. Seit Jahren füllten Quellenstudien über diesen Gegenstand die kargen Stunden seiner Mufse. Mit Bienenfleifs und der ihm eigenen Findigkeit ging er an die Sammlung des vorhandenen Materials, an die kritische Sichtung wissenschaftlicher Notizen. Schon die Vorarbeit, die von mir bereits erwähnte bibliographische und biographische Einleitung zum „,neuen Naumann", enthält eine Fülle verblüffend neuer Facten und Gesichtspunkte, mit denen Leverkühn den literarischen Kennern und Feinschmeckern aufwartete. Diese Schrift, in einem wundervollen, mit Facsimile-Drucken ausgestatteten Separatdruck ${ }^{2}$ ) erschienen, stellt ein anziehendes bibliographisches Verzeichnis dar, mit seinem Sprudel literarhistorischer Bemerkungen. Es ist kein oberflächliches, amüsantes Compendium zum bequemen Blättern, es gibt vielmehr eine entzückende Menge von Details und positiven Tatsachen. Nach der Veröffentlichung jener Arbeit hatte Leverkühn

1) H. Schalow und Léon Olphe-Galliard, Jean Crespon. Eine biographische Skizze; Ornith. Centralblatt, 1880, No. 14, 103-108.

2) Paul Leverkühn, Biographisches über die drei Naumanns und Bibliographisches über ibre Werke nebst den Vorreden zur zweiten Auflage der Naturgeschichte der $\nabla o ̈ g e l$ Deutschlands. Mit 8 nur für diesen Sonderdruck hergestellten Tafeln. Gera-Untermhaus $1 / 2$ gr. fol. 1904, 88 pp. 
noch in Ziebigk geweilt und an Ort und Stelle, unterstüzt von Frau Amtmann Elise Naumann, seine Quellenstudien getrieben.1 Mancherlei handschriftliches Material war ihm durch die Hände gegangen und manches davon ihm zu erwerben gelungen. Jacob Moyat in Mainz, der bedeutendste lebende Naumann Kenner und beneidenswerte Besitzer der reichsten Bibliothek Naumann'scher Veröffentlichungen, hatte Leverkühn in vorbildlicher Uneigennützigkeit bei der Beschaffung von Material unterstützt und die schwer zu erhaltenden Porträts zeitgenössischer Ornithologen Naumanns, z. B. von Bruch in Mainz, Meyer in Offenbach, Wolf in Nürnberg - oft auf schwierigen Wegen - für die zu veröffentlichende Naumann Biographie beschafft. So war denn die Aussicht vorhanden, eine zusammenhängende Darstellung des Lebensganges unseres grofsen Joh. Fr. Naumann zu erhalten. Wir durften überzeugt sein, das nach der Vollendung des Werkes eine ausgezeichnete Leistung vorliegen würde. Es ist Leverkühn, wie ich aus seinen persönlichen Mitteilungen schliefsen darf, gelungen, den berühmten deutschen Ornithologen in seiner wissenschaftlichen Bedeutung zu erfassen und ihn als Menschen in harmonischem Zusammenklang seines Wesens zu schildern. Weit über die Bedeutung hinaus, die der Darstellung des Lebens eines einzelnen Menschen beigemessen werden kann, dürfte das Werk Leverkühns für uns wachsen, weil sich in der geschilderten Persönlichkeit Naumann's die Entwickelung unserer ornithologischen Biologie wiederspiegelt. In frühem Alter - in noch nicht vollendetem 39. Lebensjahre - hat Leverkühn scheiden müssen. Seine Arbeit liegt unvollendet. S. M. König Ferdinand von Bulgarien, der des Verstorbenen Pläne bezüglich derselben genau kennt, hat sich die Herausgabe des nachgelassenen Werkes vorbehalten. In keine besseren Hände könnte sie gelegt werden.

Möge diese Naumann Biographie bald erscheinen: zur Erinnerung an Paul Leverkühn und zur Ehre und zum bleibenden Gedenken an einen deutschen Gelehrten, dessen arbeitsreiches Leben fruchtbar gewesen ist und anregend und fruchtbringend für die Vogelkunde auch noch weiterhin sein wird.

1) Paul Leverkühn, The ornithological correspondence of the late Professor Johann Friedrich Naumann. (Les Correspondances ornithologiques du Professeur Fréd. Naumann, Présentation de lettres de Temminck, Boie, Brehm, Kaup, Bechstein, Gloger, Bruch, and others); Proc. Fourth Internat. Ornith. Congress London June 1905. London 1907 , gr. $8^{0}, 157-162$. 


\section{$2 \mathrm{BHL}$ Biodiversity Heritage Library}

1909. "Bericht Über den gegenwärtigen Stand der Naumannforschung." Journal $f u$

r Ornithologie 57, 49-55. https://doi.org/10.1007/bf02094648.

View This Item Online: $\underline{\text { https://www.biodiversitylibrary.org/item/43500 }}$

DOI: https://doi.org/10.1007/bf02094648

Permalink: https://www.biodiversitylibrary.org/partpdf/142387

\section{Holding Institution}

MBLWHOI Library

\section{Sponsored by}

MBLWHOI Library

\section{Copyright \& Reuse}

Copyright Status: No known copyright restrictions as determined by scanning institution.

This document was created from content at the Biodiversity Heritage Library, the world's largest open access digital library for biodiversity literature and archives. Visit BHL at https://www.biodiversitylibrary.org. 